MATHEMATICS OF COMPUTATION

Volume 70, Number 235, Pages 1031-1042

S 0025-5718(00)01266-7

Article electronically published on June 12, 2000

\title{
BOUNDARY ELEMENT METHODS \\ FOR POTENTIAL PROBLEMS WITH NONLINEAR BOUNDARY CONDITIONS
}

\author{
M. GANESH AND O. STEINBACH
}

Dedicated to Professor Ian Sloan on the occasion of his 60th birthday

\begin{abstract}
Galerkin boundary element methods for the solution of novel first kind Steklov-Poincaré and hypersingular operator boundary integral equations with nonlinear perturbations are investigated to solve potential type problems in two- and three-dimensional Lipschitz domains with nonlinear boundary conditions. For the numerical solution of the resulting Newton iterate linear boundary integral equations, we propose practical variants of the Galerkin scheme and give corresponding error estimates. We also discuss the actual implementation process with suitable preconditioners and propose an optimal hybrid solution strategy.
\end{abstract}

\section{INTRODUCTION}

In this work, we are interested in the application of Galerkin boundary element methods for the numerical solution of the nonlinear boundary integral equations

$$
\begin{gathered}
(S u)(x)+(N u)(x)=f(x) \quad \text { for } x \in \Gamma, \\
(D v)(x)+\left[N\left(\frac{1}{2} I-K\right) v\right](x)=f(x) \quad \text { for } x \in \Gamma,
\end{gathered}
$$

where $S$ is the Steklov-Poincaré operator

$$
(S u)(x)=\left[D+\left(\frac{1}{2} I+K^{\prime}\right) V^{-1}\left(\frac{1}{2} I+K\right)\right] u(x) .
$$

In (1.1)-(1.3) $, V, K, K^{\prime}$ and $D$ are, respectively, boundary integral operators on a Lipschitz boundary $\Gamma$ of the single, double, adjoint of the double, and the hypersingular layer potentials [6] 12, and $N$ is the Nemytskii operator $(N u)(x)=$ $g(x, u(x)), x \in \Gamma$.

The nonlinear boundary integral equations (1.1) and (1.2) are equivalent to a boundary value problem in a bounded domain $\Omega \subset \mathbb{R}^{n}(n=2,3)$ with Lipschitz

Received by the editor September 10, 1998 and, in revised form, November 3, 1998 and July 30, 1999.

2000 Mathematics Subject Classification. Primary 31C20, 65L20, 65N38, 74S15.

Key words and phrases. Boundary element methods, Nonlinear boundary conditions.

Part of this work was carried out while the second author was a Visiting Fellow in the School of Mathematics, UNSW, under an Australian Research Council Grant. The support of the Australian Research Council is gratefully acknowledged by both authors.

(C)2000 American Mathematical Society 
boundary $\Gamma$, and a nonlinear boundary condition

$$
\mathcal{E} u(x)=0 \quad \text { for } x \in \Omega, \quad(T u)(x)+g(x, u(x))=f(x) \quad \text { for } x \in \Gamma .
$$

Here, $\mathcal{E}$ is a second order linear partial differential operator and $T$ is the corresponding conormal derivative operator. We assume that there is given a fundamental solution of $\mathcal{E}$ implying all the boundary integral operators $V, K, K^{\prime}$ and $D$. In general we require only that the resulting single layer potential $V$ is $H^{-1 / 2}(\Gamma)$-elliptic, the hypersingular integral operator $D$ is $H^{1 / 2}(\Gamma)$-semi-elliptic and the double layer potential $K$ satisfies the properties as in [12. Our results in [12] demonstrating the equivalence between the boundary value problem (1.4) and the nonlinear boundary integral equations (1.1) and (1.2) for the Laplace operator case then remains true for the above general situation as well. The first equivalent formulation is obtained through the Dirichlet-Neumann map and (1.2) follows from an indirect double layer potential ansatz. The properties we need on boundary integral operators are valid for many general situations and throughout the paper; we use the results in [12].

The model (1.4) includes the classical Robin problem and nonlinear boundary conditions occur for example in steady-state heat diffusion equations where the heat flux across a blackbody surface $\Gamma$ based on the Stefan-Boltzman law is given by a nonlinear function of the temperature $u$, typically $O\left(u^{4}\right)$, 14, p. 10, 57] (see also [3, p. 219], 15, p. 419], 16, p. 248]). Other examples of such a model are for contact problems in linear elasticity, with and without friction (see, for example, $[8$ and references therein), and in fracture mechanics based on minimising certain energy functional on a domain of harmonic functions satisfying a nonlinear boundary condition [11. Note that in these and other applications, $u, g$ and $f$ may be vector valued, and may involve an additional simple Dirichlet boundary condition. These additional features can be incorporated in our analysis, depending on the problem, by introducing appropriate product spaces and suitable matrix version of the above equations. Our aim in this paper, however, is to describe in detail numerical solvability of (1.1) and (1.2), and we hope it will have a good spin-off for many applications in future work, including BEM-FEM coupling [4. As in [12] we further assume

(A1) $f \in L^{2}(\Gamma)$ and (1.4) has an isolated solution $u \in H^{1+s}(\Omega)$ for some $s \geq \frac{1}{2}$.

(A2) For all $x \in \Gamma, g(x, \cdot): \mathbb{R} \rightarrow \mathbb{R}$ is twice differentiable and the derivatives are locally bounded, i.e., for every finite interval $[a, b]$, there exists a constant $M_{[a, b]}$ such that $\left|\frac{\partial^{i} g(x, \alpha)}{\partial^{i} \alpha}\right| \leq M_{[a, b]}$ for $x \in \Gamma, a \leq \alpha \leq b, i=1,2$.

Since we require $f$ in (1.4) to be in $L^{2}(\Gamma)$, it is natural to assume $s \geq \frac{1}{2}$ in (A1). However, our error analysis goes through for all $s>0$ yielding corresponding lower convergence rates. In [12, using (A1)-(A2), we proved that (1.1) and (1.2) have isolated solutions in $H^{1}(\Gamma)$, denoted by $u^{*}$ and $v^{*}$, respectively. The regularity of the solutions $u^{*}, v^{*}$ is restricted to $H^{1}(\Gamma)$ only in the Lipschitz boundary case. However, if $\Gamma \subset \mathbb{R}^{2}$ is a piecewise $C^{\infty}$ boundary, given as a union of locally smooth parts $\Gamma^{j}$, we have $u^{*} \in H^{1 / 2+s}(\Gamma)$ with $s \geq \frac{1}{2}$ as in (A1), but $v^{*} \in H^{1 / 2+\sigma}(\Gamma)$ only for $\sigma \in\left(-\sigma_{0}, \sigma_{0}\right)$ with $\sigma_{0}$ determined by the interior angles [12. If $\omega_{j}$ is the interior angle at the $j$ th corner point, then $\sigma_{0}:=\min _{j=1, \ldots, J}\left\{\sigma_{j}\right\}$, with $\sigma_{j}:=\min \left\{\frac{\pi}{\omega_{j}}, \frac{\pi}{2 \pi-\omega_{j}}\right\}$. Due to this regularity restriction and since (1.2) is computationally more efficient than (1.1), we will propose a hybrid solution strategy by suitably combining both the formulations. 
Earlier work in solving (1.4) was based on a direct boundary integral formulation initiated in [18] and further studied in [1, 7, 9, 10] 17]. In the direct formulation the nonlinear operator appears as density of the double layer potential, adding additional difficulty in dealing with the nonlinearity. In our formulations (11.1) and (1.2) the nonlinearity does not appear as a density of boundary integral operators. We give complete error analysis of some practically computable approximations to the solution of (1.1) and (1.2). The convergence rates obtained in this work are similar to those obtained in [18] and in the sequel work. But, earlier results cover only the two-dimensional case while results in our work are for two- and three-space problems with Lipschitz boundaries.

Further, all the earlier research involves discretizing the nonlinear problem first, leading to solving the resulting nonlinear algebraic systems based on some blackbox routine. So no error analysis in solving these systems was given; the matrix structure and conditioning of the associated linearised system was not investigated. In contrast, our methods and analysis in this work give a clear picture of the entire approximation process in computing approximate solutions of (1.1) and (1.2) and show exact representations of the matrix structures involved. Hence, based on the boundary integral operators in our formulations, we find suitable preconditioners needed for efficiently solving the linear systems. All these naturally involve some preliminary work, so that the entire computational procedure is somewhat within our control rather than based on a complicated general-purpose black-box nonlinear algebraic solver.

In this paper, compared to all earlier work discussed above, we follow an entirely different approach. We first apply the standard Newton scheme to the continuous problems and show quadratic convergence of the Newton iterates. Following [12], the Newton iterates for (1.1) and (1.2), respectively, for $k=0,1, \ldots$ are

$$
\left[S+N^{\prime}\left(u^{k}\right)\right] u^{k+1}=f+N^{\prime}\left(u^{k}\right) u^{k}-N u^{k}
$$

and

$$
\left[D+N^{\prime}\left(u^{k}\right)\left(\frac{1}{2} I-K\right)\right] v^{k+1}=f+N^{\prime}\left(u^{k}\right) u^{k}-N u^{k},
$$

where in (1.6), $u^{k}=\left(\frac{1}{2} I-K\right) v^{k}$. We start with $u^{0}, v^{0}$ with $u^{0} \in \mathcal{U}_{\rho}\left(u^{*}\right)$, where $u^{*}$ is the trace of the isolated solution of (1.4), and $\mathcal{U}_{\rho}\left(u^{*}\right)$ is a ball in $H^{1 / 2}(\Gamma)$ with centre $u^{*}$ and radius $\rho$. We use the standard notation $N^{\prime}\left(u^{k}\right)$ for the Fréchet derivative of $N$ at $u^{k}$. If $\rho$ is sufficiently small, then in [12] we proved that for all $k \geq 0$, (1.5) and (1.6) have unique solutions $u^{k+1}$ and $v^{k+1}$ in $H^{1}(\Gamma)$ (with $\left.u^{k+1} \in \mathcal{U}_{\rho}\left(u^{*}\right)\right)$ and converge quadratically to $u^{*}$ and $v^{*}$, respectively. Henceforth, we assume that $\rho$ is small enough so that $u^{k+1} \in \mathcal{U}_{\rho}\left(u^{*}\right)$. The stopping criteria for the Newton algorithm involves calculating the residuum $r^{k+1}$ using a modified formula, not involving boundary integral operators [12]:

$$
r^{k+1}=N^{\prime}\left(u^{k}\right)\left(u^{k}-u^{k+1}\right)+N u^{k+1}-N u^{k} .
$$

Due to the above algorithm and the corresponding convergence result, to solve (1.1) and (1.2) it is enough to concentrate on computing solutions of (1.5) and (1.6).

We organise the rest of the paper as follows. Practical variants of the Galerkin method are proposed and analysed for the resulting sequence of linear problems in Section 2. In Section 3 a preconditioning strategy for solving the linear systems is given, and we conclude the paper in Section 4 with numerical examples. 
Throughout the paper, $c$ will denote a general constant which may have different values at different occurrences and is independent of the boundary element discretization parameter $h$ and the Newton iteration index $k$.

\section{GALERKin APPROXIMATION SCHEMES}

In this section we study practical variants of the Galerkin method to compute solutions of (1.5) and (1.6) at each step of the Newton iteration.

For this purpose we define a family of trial spaces $W_{h}=\operatorname{span}\left\{\varphi_{j}^{\mu}\right\}_{j=1}^{M} \subset H^{1 / 2}(\Gamma)$ of $B$-splines of (piecewise polynomial) degree $\mu$ satisfying the approximation property

$$
\inf _{w_{h} \in W_{h}}\left\|w-w_{h}\right\|_{H^{\tau}(\Gamma)} \leq c \cdot h^{\sigma-\tau} \cdot\|w\|_{H^{\sigma}(\Gamma)}
$$

for all $w \in H^{\sigma}(\Gamma)$ with $\tau \leq \sigma \leq \mu+1$ and $\tau<\mu+\frac{1}{2}(n=2) ; \tau \leq \mu(n=3)$. (For example we can use piecewise linear continuous hat functions, i.e., $\mu=1$, defined over a boundary element mesh on $\Gamma$ with mesh size $h$.) Note that the approximation property (2.1) requires only a regular triangulation, i.e., we allow adaptive refinements and appropriate nonuniform meshes. In this work we do not include approximation of the boundary. The definition of $W_{h}$ may depend on the Newton iteration index $k$ to solve the linearised system. This means that we can adapt $W_{h}$ during the Newton iteration process and hence we denote $h_{k}$ to be the mesh size of the triangulation at the $k$ th Newton step.

The Steklov-Poincaré operator formulation. First consider the Galerkin variational formulation of (1.5). Find $u_{h}^{k+1} \in W_{h}$ such that

$$
\left\langle\left[S+N^{\prime}\left(u^{k}\right)\right] u_{h}^{k+1}, v_{h}\right\rangle=\left\langle\tilde{f}\left(u^{k}\right), v_{h}\right\rangle
$$

is satisfied for all test functions $v_{h} \in W_{h}$ where

$$
\tilde{f}(\varphi):=f+N^{\prime}(\varphi) \varphi-N \varphi, \quad \varphi \in H^{1 / 2}(\Gamma) .
$$

Since in practical computations we have to replace in (2.2) the Steklov-Poincaré operator $S$ as given in (1.3) by some suitable approximation $\widetilde{S}$, and $u^{k}$ by the previous approximation, we solve instead the modified Galerkin variational problem: find $\hat{u}_{h}^{k+1} \in W_{h}$ such that

$$
\left\langle\left[\widetilde{S}+N^{\prime}\left(\hat{u}_{h}^{k}\right)\right] \hat{u}_{h}^{k+1}, v_{h}\right\rangle=\left\langle\tilde{f}\left(\hat{u}_{h}^{k}\right), v_{h}\right\rangle \quad \text { for all } v_{h} \in W_{h} .
$$

To introduce a computable approximation $\tilde{S}$ we proceed as follows. For an arbitrary but fixed $v \in H^{1 / 2}(\Gamma)$, from (1.3) we have $S v=D v+\left(\frac{1}{2} I+K^{\prime}\right) w$, where $w$ is the unique solution of the variational problem

$$
\langle V w, \tau\rangle=\left\langle\left(\frac{1}{2} I+K\right) v, \tau\right\rangle \quad \text { for all } \tau \in H^{-1 / 2}(\Gamma) .
$$

Let

$$
Z_{h}=\operatorname{span}\left\{\varphi_{j}^{\nu}\right\}_{j=1}^{N} \subset H^{-1 / 2}(\Gamma)
$$

be some trial space satisfying the approximation property in $H^{-1 / 2}(\Gamma)$. For example, we can take $Z_{h}$ to be the space of piecewise constant trial functions (i.e., 
$\nu=0$ ). Now we consider the Galerkin variational problem of (2.5): find $w_{h} \in Z_{h}$ satisfying

$$
\left\langle V w_{h}, \tau_{h}\right\rangle=\left\langle\left(\frac{1}{2} I+K\right) v, \tau_{h}\right\rangle \quad \text { for all } \tau_{h} \in Z_{h} .
$$

Hence, we may define an approximation of the Steklov-Poincaré operator by

$$
\tilde{S} v:=D v+\left(\frac{1}{2} I+K^{\prime}\right) w_{h}
$$

It is easy to see that

$$
\|(S-\tilde{S}) v\|_{-1 / 2} \leq c \cdot\left\|w-\tilde{w}_{h}\right\|_{-1 / 2} \leq c \cdot \inf _{\tau_{h} \in Z_{h}}\left\|w-\tau_{h}\right\|_{-1 / 2} .
$$

Using the Aubin-Nitsche trick [13] we get

$$
\|(S-\tilde{S}) v\|_{-1} \leq c \cdot h^{1 / 2} \cdot\left\|w-w_{h}\right\|_{-1 / 2} .
$$

The variational problem (2.4) is equivalent to the system of linear equations

$$
\left[D_{h}+\left(\frac{1}{2} I_{h}^{\top}+K_{h}^{\top}\right) V_{h}^{-1}\left(\frac{1}{2} I_{h}+K_{h}\right)+N_{h}^{\prime}\left(\hat{u}_{h}^{k}\right)\right] \underline{u}^{k+1}=\underline{f},
$$

where for $i, j=1, \ldots, M ; p, q=1, \ldots, N$,

$$
\begin{aligned}
D_{h}[j, i] & =\left\langle D \varphi_{i}^{\mu}, \varphi_{j}^{\mu}\right\rangle, & I_{h}[q, i] & =\left\langle\varphi_{i}^{\mu}, \varphi_{q}^{\nu}\right\rangle, \\
V_{h}[q, p] & =\left\langle V \varphi_{p}^{\nu}, \varphi_{q}^{\nu}\right\rangle, & N_{h}^{\prime}\left(\varphi_{h}\right)[j, i] & =\left\langle N^{\prime}\left(\varphi_{h}\right) \varphi_{i}^{\mu}, \varphi_{j}^{\mu}\right\rangle, \\
K_{h}[q, i] & =\left\langle K \varphi_{i}^{\mu}, \varphi_{q}^{\nu}\right\rangle, & f_{j} & =\left\langle\tilde{f}\left(\hat{u}_{h}^{k}\right), \varphi_{j}^{\mu}\right\rangle .
\end{aligned}
$$

To solve (2.11), we need the inverse matrix of the discrete single-layer potential $V_{h}$. This corresponds to a solution of a linear system $V_{h} \underline{w}=\underline{r}$ per iteration step. For this one may perform an LU decomposition of $V_{h}$ in advance only once, which will be asymptotically of order $O\left(N^{3}\right)$, or use again a preconditioned inner iterative scheme [2], say $c g$, with some preconditioner as proposed in 21] of order $O\left(N^{2}\right)$ only, with an appropriate stopping criteria as given in Section 4.

Our immediate task is to establish the stability and convergence of the solutions $\hat{u}_{h}^{k+1}$ of the variational problem (2.4) to the solutions $u^{k+1}$ of (1.5) at each Newton iteration step. For this reason, it is theoretically useful to consider the additional problem: find $\hat{u}^{k+1} \in H^{1 / 2}(\Gamma)$ satisfying

$$
\left[\widetilde{S}+N^{\prime}\left(\hat{u}_{h}^{k}\right)\right] \hat{u}^{k+1}=\tilde{f}\left(\hat{u}_{h}^{k}\right) .
$$

Note that (2.12) corresponds to (1.5) with an approximated operator and an approximated right-hand side, while (2.4) is the Galerkin variational formulation of (2.12). Error estimates based on the Strang lemma are already known in the case of linear perturbations [5], but in (2.12) both the approximation of the operator and the right-hand side are nonlinear.

Due to the positive definiteness of the single layer potential $V_{h}$, we have

$$
\left\langle D v_{h}, v_{h}\right\rangle \leq\left\langle\tilde{S} v_{h}, v_{h}\right\rangle \text { for all } v_{h} \in W_{h} .
$$

Using this, the positive definiteness of $D+L$ where

$$
(L u)(x)=\int_{\Gamma} u(y) d s_{y} \quad \text { for } u \in H^{1 / 2}(\Gamma), \quad x \in \Gamma,
$$

and (A1)-(A2), we get the following properties (with $\varphi \in\left\{u^{k}, \hat{u}_{h}^{k}\right\} \subset \mathcal{U}_{\rho}\left(u^{*}\right)$ ): 
(P1) $\widetilde{S}+L: H^{1 / 2}(\Gamma) \rightarrow H^{-1 / 2}(\Gamma)$ is a linear bounded and positive definite operator.

(P2) $N^{\prime}(\varphi)-L: L^{2}(\Gamma) \rightarrow H^{\alpha}(\Gamma)$ is bounded for $\alpha=0$, and compact for $\alpha=-1 / 2$.

(P3) The linear form $\langle\tilde{f}(\varphi), \cdot\rangle$ is bounded in $H^{1 / 2}(\Gamma)$.

(P4) $\widetilde{S}+N^{\prime}(\varphi): H^{1 / 2}(\Gamma) \rightarrow H^{-1 / 2}(\Gamma)$ is a linear and bounded operator satisfying a Gårdings inequality, i.e., there exist positive constants $c_{2}, c_{3}, c_{4}$, such that

$$
\begin{aligned}
& \operatorname{Re}\left(\left\langle\left(\widetilde{S}+N^{\prime}(\varphi)\right) u, u\right\rangle\right) \geq c_{2} \cdot\|u\|_{1 / 2}^{2}-c_{3} \cdot\|u\|_{0}^{2} \quad \text { for all } u \in H^{1 / 2}(\Gamma) \\
& \left|\left\langle\left(\widetilde{S}+N^{\prime}(\varphi)\right) u, v\right\rangle\right| \leq c_{4} \cdot\|u\|_{1 / 2}\|v\|_{1 / 2} \quad \text { for all } u, v \in H^{1 / 2}(\Gamma) .
\end{aligned}
$$

We first prove the convergence of the modified Galerkin solutions $\hat{u}_{h}^{k+1}$ of (2.4) to $u^{k+1}$.

Theorem 2.1. Let (A0)-(A2) be satisfied. Let $\hat{u}_{h}^{0}=u^{0} \in W_{h} \cap \mathcal{U}_{\rho}\left(u^{*}\right)$ with $\rho$ sufficiently small. Then there exists an $h_{0}>0$ such that for all $k \geq 0$ and all $h \in\left(0, h_{0}\right)$, (2.4) has a unique solution $\hat{u}_{h}^{k+1} \in W_{h}$ with error estimate

$$
\begin{aligned}
\left\|u^{k+1}-\hat{u}_{h}^{k+1}\right\|_{1 / 2} \leq c \cdot\left\{\inf _{w_{h} \in W_{h}}\right. & \left\|u^{k+1}-w_{h}\right\|_{1 / 2} \\
& \left.+\left\|u^{k}-\hat{u}_{h}^{k}\right\|_{0}+\left\|(S-\tilde{S}) u^{k+1}\right\|_{-1 / 2}\right\} .
\end{aligned}
$$

Further, for all $k \geq 0$,

$$
\left\|u^{k+1}-\hat{u}_{h}^{k+1}\right\|_{1 / 2} \leq c\left\{h_{k+1}^{1 / 2}\left\|u^{k+1}\right\|_{1}+\left\|u^{k}-\hat{u}_{h}^{k}\right\|_{0}+\left\|(S-\tilde{S}) u^{k+1}\right\|_{-1 / 2}\right\}
$$

and, if $\nu=\mu-1$,

$$
\left\|u^{k+1}-\hat{u}_{h}^{k+1}\right\|_{1 / 2} \leq c \cdot \sum_{\ell=0}^{k}\left\{h_{\ell+1}^{1 / 2} \cdot\left\|u^{\ell+1}\right\|_{1}\right\} \rightarrow 0 \quad \text { as } h_{\ell} \rightarrow 0 \forall \ell .
$$

Proof. We will prove the result in five steps. Let $k=0$.

1. Since $\hat{u}^{k}, \hat{u}_{h}^{k} \in \mathcal{U}_{\rho}\left(u^{*}\right)$, by [12, Theorem 3.2] both equations (1.5) and (2.12) are uniquely solvable. The injectivity of $\tilde{S}+N^{\prime}\left(\hat{u}_{h}^{k}\right)$, Gårdings inequality (2.15) and (P1)-(P4) yield (see [20]) that there exists an $h_{0}^{k}>0$ such that for all $h \in\left(0, h_{0}^{k}\right)$ (2.4) has a unique solution $\hat{u}_{h}^{k+1} \in W_{h}$ and $\left\|\hat{u}^{k+1}-\hat{u}_{h}^{k+1}\right\|_{1 / 2} \leq$ $c \inf _{w_{h} \in W_{h}}\left\|\hat{u}^{k+1}-w_{h}\right\|_{1 / 2}$. Using this inequality, we get

$$
\left\|u^{k+1}-\hat{u}_{h}^{k+1}\right\|_{1 / 2} \leq(1+c)\left\|u^{k+1}-\hat{u}^{k+1}\right\|_{1 / 2}+c \inf _{w_{h} \in W_{h}}\left\|u^{k+1}-w_{h}\right\|_{1 / 2}
$$

Hence to show (2.16) it is sufficient to bound $\left\|u^{k+1}-\hat{u}^{k+1}\right\|_{1 / 2}$.

2. From (2.12) and (1.5) we have

$$
\begin{aligned}
{\left[\widetilde{S}+N^{\prime}\left(\hat{u}_{h}^{k}\right)\right] \hat{u}^{k+1} } & =\tilde{f}\left(\hat{u}_{h}^{k}\right) \\
{\left[\widetilde{S}+N^{\prime}\left(\hat{u}_{h}^{k}\right)\right] u^{k+1} } & =\tilde{f}\left(u^{k}\right)+\left[N^{\prime}\left(\hat{u}_{h}^{k}\right)-N^{\prime}\left(u^{k}\right)\right] u^{k+1}+[\widetilde{S}-S] u^{k+1} .
\end{aligned}
$$


The bounded inverse of $\widetilde{S}+N^{\prime}\left(\hat{u}_{h}^{k}\right): H^{1 / 2}(\Gamma) \rightarrow H^{-1 / 2}(\Gamma)$ and (2.3) yield

$$
\begin{aligned}
\left\|u^{k+1}-\hat{u}^{k+1}\right\|_{1 / 2} \leq & c\left\|\tilde{f}\left(u^{k}\right)-\tilde{f}\left(\hat{u}_{h}^{k}\right)+\left[N^{\prime}\left(\hat{u}_{h}^{k}\right)-N^{\prime}\left(u^{k}\right)\right] u^{k+1}\right\|_{0} \\
& +\left\|(\widetilde{S}-S) u^{k+1}\right\|_{-1 / 2} \\
\leq & c\left\{\left\|N^{\prime}\left(u^{k}\right) u^{k}-N^{\prime}\left(\hat{u}_{h}^{k}\right) \hat{u}_{h}^{k}\right\|_{0}+\left\|N u^{k}-N \hat{u}_{h}^{k}\right\|_{0}\right. \\
& \left.\quad+\left\|\left[N^{\prime}\left(u^{k}\right)-N^{\prime}\left(\hat{u}_{h}^{k}\right)\right] u^{k+1}\right\|_{0}+\left\|(\widetilde{S}-S) u^{k+1}\right\|_{-1 / 2}\right\} .
\end{aligned}
$$

We can bound the first term by

$$
\begin{aligned}
\left\|N^{\prime}\left(u^{k}\right) u^{k}-N^{\prime}\left(\hat{u}_{h}^{k}\right) \hat{u}_{h}^{k}\right\|_{0} & \leq\left\|\left[N^{\prime}\left(u^{k}\right)-N^{\prime}\left(\hat{u}_{h}^{k}\right)\right] u^{k}\right\|_{0}+\left\|N^{\prime}\left(\hat{u}_{h}^{k}\right)\left[u^{k}-\hat{u}_{h}^{k}\right]\right\|_{0} \\
& \leq c \cdot\left\|u^{k}-\hat{u}_{h}^{k}\right\|_{0},
\end{aligned}
$$

where we have used the boundedness of $N^{\prime}\left(\hat{u}_{h}^{k}\right)$ on $L^{2}(\Gamma)$, and the following bound obtained by the mean value theorem, (A2) and $u^{k} \in \mathcal{U}_{\rho}\left(u^{*}\right)$ :

$$
\begin{aligned}
\left|\left[N^{\prime}\left(u^{k}\right)-N^{\prime}\left(\hat{u}_{h}^{k}\right)\right] u^{k}(x)\right| & =\left|g_{\alpha}\left(x, u^{k}(x)\right)-g_{\alpha}\left(x, \hat{u}_{h}^{k}(x)\right)\right| \cdot\left|u^{k}(x)\right| \\
= & \left|u^{k}(x)-\hat{u}_{h}^{k}(x)\right| \cdot\left|g_{\alpha \alpha}(x, \eta(x))\right| \cdot\left|u^{k}(x)\right| \leq c \cdot\left|u^{k}(x)-\hat{u}_{h}^{k}(x)\right|
\end{aligned}
$$

with $\eta(x)$ between $u^{k}(x)$ and $\hat{u}_{h}^{k}(x)$. Using (A2) and the above arguments,

$\left\|N u^{k}-N \hat{u}_{h}^{k}\right\|_{0} \leq c \cdot\left\|u^{k}-\hat{u}_{h}^{k}\right\|_{0}, \quad\left\|\left[N^{\prime}\left(u^{k}\right)-N^{\prime}\left(\hat{u}_{h}^{k}\right)\right] u^{k+1}\right\|_{0} \leq c \cdot\left\|u^{k}-\hat{u}_{h}^{k}\right\|_{0}$.

Hence we have from (2.20),

$$
\left\|u^{k+1}-\hat{u}^{k+1}\right\|_{1 / 2} \leq c \cdot\left\{\left\|u^{k}-\hat{u}_{h}^{k}\right\|_{0}+\left\|(\widetilde{S}-S) u^{k+1}\right\|_{-1 / 2}\right\} .
$$

3. Using (2.21) in (2.19) gives (2.16). From [12, Theorem 3.2], $u^{k+1} \in H^{1}(\Gamma)$. Using the approximation property (2.1) (with $\tau=\frac{1}{2}, \sigma=1$ ) in (2.16) we get the inequality (2.17). Using $\hat{u}_{h}^{0}=u^{0}$ and the approximation property of $Z_{h}$ (with $\nu=\mu-1$ ), we get (2.18) from (2.17).

4. Since $\rho$ is sufficiently small, from [12, Theorem 3.2] $u^{k+1} \in \mathcal{U}_{\rho}\left(u^{*}\right)$. So using

$$
\left\|u^{*}-\hat{u}_{h}^{k+1}\right\|_{1 / 2} \leq\left\|u^{*}-u^{k+1}\right\|_{1 / 2}+\left\|u^{k+1}-\hat{u}_{h}^{k+1}\right\|_{1 / 2},
$$

for sufficiently small $h, u^{k+1}, \hat{u}_{h}^{k+1} \in \mathcal{U}_{\rho}\left(u^{*}\right)$.

5. If we replace $k$ by $k+1$ and repeat steps 1 to 4 above, we get the result for all $k \geq 0$, with $h_{0}=\min _{\ell=0, \ldots, k} h_{0}^{\ell}$.

As in Theorem 2.1, henceforth we take $\nu=\mu-1$ in (2.6). For a general Lipschitz boundary $\Gamma, u^{k+1} \in H^{1}(\Gamma)$ only and hence we have a maximal order of convergence $\frac{1}{2}$. However, in case of a (piecewise) $C^{\infty}$ boundary $\Gamma$ we have $u^{*} \in H^{1 / 2+s}$ for some $s \geq 1 / 2$ given by (A1). Hence, for a polygonal boundary $\Gamma$ and a sufficiently regular solution $u^{*}$ we can formulate the following convergence result.

Corollary 2.1. Under the assumptions and details as in Theorem 2.1,

$$
\begin{array}{r}
\left\|u^{*}-\hat{u}_{h}^{k+1}\right\|_{1 / 2} \leq c\left\{\inf _{w_{h} \in W_{h}}\left\|u^{*}-w_{h}\right\|_{1 / 2}+\left\|u^{*}-u^{k+1}\right\|_{1 / 2}\right. \\
\left.+\left\|u^{k}-\hat{u}_{h}^{k}\right\|_{0}+\left\|(S-\tilde{S}) u^{k+1}\right\|_{-1 / 2}\right\} .
\end{array}
$$

If $u^{*} \in H^{\sigma}(\Gamma)$, then for all $s$ with $\frac{1}{2} \leq s \leq \min \{\sigma, \mu+1\}$,

$$
\left\|u^{*}-\hat{u}_{h}^{k+1}\right\|_{1 / 2} \leq c \cdot\left\{h_{k+1}^{s-\frac{1}{2}} \cdot\left\|u^{*}\right\|_{s}+\left\|u^{k}-\hat{u}_{h}^{k}\right\|_{0}+\rho^{k}\right\} .
$$


In (2.24), the term $\rho^{k}$ is obtained using the quadratic convergence of the Newton iterates [12, Theorem and Remark 3.2] and the fact that $u^{k+1} \in \mathcal{U}_{\rho}\left(u^{*}\right)$ for all $k$. Applying the Aubin-Nitsche trick, we get the following error estimates in $L^{2}(\Gamma)$.

Corollary 2.2. Under the same assumptions and details as in Theorem 2.1,

$$
\left\|u^{k+1}-\hat{u}_{h}^{k+1}\right\|_{0} \leq c \cdot\left\{h_{k+1}^{1 / 2} \cdot\left\|u^{k+1}-\hat{u}_{h}^{k+1}\right\|_{1 / 2}+\left\|\hat{u}_{h}^{k}-u^{k}\right\|_{0}\right\} .
$$

If $u^{*} \in H^{\sigma}(\Gamma)$, then for all $s$ with $\frac{1}{2} \leq s \leq \min \{\sigma, \mu+1\}$,

$$
\left\|u^{*}-\hat{u}_{h}^{k+1}\right\|_{0} \leq c \cdot\left\{h_{k+1}^{s} \cdot\left\|u^{*}\right\|_{s}+\left\|u^{k}-\hat{u}_{h}^{k}\right\|_{0}+\rho^{k}\right\} .
$$

The indirect hypersingular integral formulation. The modified Galerkin method we propose to solve the linearised equation (1.6) for the indirect hypersingular integral formulation is to find $\hat{v}_{h}^{k+1} \in W_{h}$ such that

$$
\left\langle\left[D+N^{\prime}\left(\hat{u}_{h}^{k}\right)\right] \hat{v}_{h}^{k+1}, w_{h}\right\rangle=\left\langle\tilde{f}\left(\hat{u}_{h}^{k}\right), w_{h}\right\rangle
$$

is satisfied for all $w_{h} \in W_{h}$, where $\hat{u}_{h}^{k} \in W_{h}$ is the solution of the variational problem

$$
\left\langle\hat{u}_{h}^{k}, w_{h}\right\rangle=\left\langle\left(\frac{1}{2} I-K\right) \hat{v}_{h}^{k}, w_{h}\right\rangle \quad \text { for all } w_{h} \in W_{h} .
$$

This is equivalent to the linear system

$$
\left[D_{h}+N_{h}^{\prime}\left(\bar{I}_{h}^{-1}\left(\frac{1}{2} \bar{I}_{h}-\bar{K}_{h}\right) \underline{v}^{k}\right)\right] \underline{v}^{k+1}=\underline{f},
$$

where, in addition to the definitions of $D_{h}, N_{h}^{\prime}\left(\varphi_{h}\right), \underline{f}$ introduced earlier, we let

$$
\bar{I}_{h}[j, i]=\left\langle\varphi_{i}^{\mu}, \varphi_{j}^{\mu}\right\rangle, \quad \bar{K}_{h}[j, i]=\left\langle K \varphi_{i}^{\mu}, \varphi_{j}^{\mu}\right\rangle \quad i, j=1, \ldots, M .
$$

Using the same arguments as in the Steklov-Poincaré operator formulation, we can prove all the above results with $u$ and $\hat{u}_{h}^{k+1}$ replaced by $v$ and $\hat{v}_{h}^{k+1}$, respectively (without the occurence of the last term in (2.16), (2.17), and (2.23)), and $\sigma$ in Corollary 2.1 replaced by the restricted quantity $\sigma_{0}$ defined in the Introduction. This is due to the fact that for a polygonal bounded boundary $\Gamma$, using the restricted mapping properties of the double layer potential operator, we get $v^{*} \in H^{1 / 2+\sigma_{0}}(\Gamma)$ only.

So, the Steklov-Poincaré operator formulation will give the best a priori error estimates as outlined in Corollary 2.1, while the hypersingular integral formulation has some restrictions. On the other hand, compared to the hypersingular integral formulation, the solution of the linearised problem in the Steklov-Poincaré operator formulation is expensive due to inversion of the single layer potential. This leads to

A hybrid solution strategy. To find an almost optimal algorithm, we want to combine the optimal error estimates of the Steklov-Poincaré operator formulation and the fast solution process of the indirect hypersingular integral formulation.

Our hybrid strategy is to first use the indirect hypersingular formulation to compute the solution to a good accuracy and then use the resulting solution as the initial guess of the Steklov-Poincaré operator formulation to compute an improved solution by solving (2.11). Due to the accuracy of the initial guess, only a few iterations of the Steklov-Poincaré operator formulation will be needed. For instance, in our numerical examples we need only one extra post-processing step. Therefore we are able to compute an approximate solution of (1.1) significantly faster. 
Error analysis of this hybrid solution strategy is nothing but the combination of the error analysis for the two formulations discussed above. Since we use an approximate solution of the indirect hypersingular integral formulation only as an initial guess for the Newton scheme, the final computed hybrid strategy solution has same the optimal convergence rates proved for the Steklov-Poincaré formulation.

\section{ItERATIVE SOLUTION STRATEGIES AND PRECONDITIONERS}

For the numerical solution of the nonlinear boundary integral equations (1.1) and (1.2) we have to solve a sequence of linear problems of the form $A_{h} \underline{v}=\underline{f}$ given by (2.11) and (2.29), respectively, at each step of the Newton iteration. Since the stiffness matrices depend on the previous Newton iterates, iterative methods are the most favourable choice to solve such linear systems, in particular we use the GMRES method [19]. Due to the order of both the Steklov-Poincaré operator and the hypersingular integral operator being one, the condition number of the stiffness matrices behave like $h^{-1}$. Hence we need to find an efficient preconditioning matrix $C_{h}$ that is spectrally equivalent to $A_{h}$ [2, 4, 19].

To this end, we first consider the matrix of the approximated Steklov-Poincaré operator. Using (2.13) and Theorem 4 in [4, it is easy to show the spectral equivalence property

$$
\left(\left(D_{h}+N_{h}^{\prime}\left(\hat{u}_{h}^{k}\right)\right) \underline{u}, \underline{u}\right) \leq\left(\left(\widetilde{S}_{h}+N_{h}^{\prime}\left(\hat{u}_{h}^{k}\right)\right) \underline{u}, \underline{u}\right) \leq c \cdot\left(\left(D_{h}+N_{h}^{\prime}\left(\hat{u}_{h}^{k}\right)\right) \underline{u}, \underline{u}\right)
$$

for all $\underline{u} \in \mathbb{R}^{M} \leftrightarrow u_{h} \in H^{1 / 2}(\Gamma)$. Hence, in both formulations of interest it is sufficient to find a preconditioner $C_{h}$ of the matrix $A_{h}:=D_{h}+B_{h}$, where we use

$$
\left(B_{h} \underline{u}, \underline{v}\right)=\left\langle B u_{h}, v_{h}\right\rangle \quad \text { for some bounded operator } B: L^{2}(\Gamma) \rightarrow L^{2}(\Gamma) .
$$

In our formulations the operator $D+B: H^{1 / 2}(\Gamma) \rightarrow H^{-1 / 2}(\Gamma)$ is injective and satisfies a Gårdings inequality. Hence it is sufficient to find a preconditioning matrix $C_{h}$ which is spectrally equivalent to $D_{h}+L_{h}$, where $L_{h}[j, i]=\left\langle L \varphi_{i}^{\mu}, \varphi_{j}^{\mu}\right\rangle$ with $L$ as defined in (2.14). In fact, following 21] such a preconditioner is $C_{h}=\bar{I}_{h} \bar{V}_{h}^{-1} \bar{I}_{h}$, where $\bar{V}_{h}[j, i]=\left\langle V \varphi_{i}^{\mu}, \varphi_{j}^{\mu}\right\rangle$ for $i, j=1, \ldots, M$ and $\bar{I}_{h}$ is as defined in (2.30)

Since results in 21 hold under minor assumptions on the triangulation of $\Gamma$, we can apply our preconditioning strategy described in this section even in the case of an adaptive refinement and appropriate nonuniform meshes. The application of the preconditioner for solving $A_{h} \underline{v}=\underline{f}$ is given by $C_{h}^{-1}=\bar{I}_{h}^{-1} \bar{V}_{h} \bar{I}_{h}^{-1}$. For this, we need two inversions of the sparse and diagonal dominant mass matrix $\bar{I}_{h}$ and one matrix times vector multiplication with $V_{h}$, which can be carried out with the same order as a multiplication with $D_{h}$ itself.

\section{Numerical Results}

In our test numerical experiment, we considered the Laplace equation and we computed solutions for both formulations described in this paper. Some important observations from the numerical experiment are that the hybrid strategy is the best to use from both computing time and accuracy points of view and that our numerical scheme is capable of allowing (in the sense of getting optimal order convergence rates) nonlinearities of the type $u^{4}$ (occuring in heat-transfer problems) that do not satisfy monotonicity assumption needed for an existence theory result in [12] (see Remark 3.1 in [12]). 
TABLE 1. Steklov-Poincaré operator formulation

\begin{tabular}{|c|c|c|c|c|c|c|}
\hline $\mathrm{N}$ & $\left\|u-u_{h}\right\|_{L^{2}(\Gamma)}$ & order & $\left\|u-u_{h}\right\|_{H^{1 / 2}(\Gamma)}$ & order & Iter & sec \\
\hline 32 & $1.35-3$ & & $7.42-3$ & & 5 & 0.37 \\
64 & $2.87-4$ & 2.23 & $2.40-3$ & 1.63 & 5 & 1.08 \\
128 & $6.43-5$ & 2.16 & $8.08-4$ & 1.57 & 5 & 3.43 \\
256 & $1.50-5$ & 2.10 & $2.78-4$ & 1.54 & 5 & 11.89 \\
512 & $3.61-6$ & 2.05 & $9.67-5$ & 1.52 & 5 & 48.91 \\
1024 & $8.86-7$ & 2.03 & $3.39-5$ & 1.51 & 5 & 198.66 \\
\hline
\end{tabular}

TABLE 2. Indirect hypersingular integral formulation

\begin{tabular}{|c|c|c|c|c|c|c|}
\hline $\mathrm{N}$ & $\left\|u-u_{h}\right\|_{L^{2}(\Gamma)}$ & order & $\left\|u-u_{h}\right\|_{H^{1 / 2}(\Gamma)}$ & order & Iter & sec \\
\hline 32 & $2.10-3$ & & $7.79-3$ & & 5 & 0.02 \\
64 & $8.15-4$ & 1.37 & $3.09-3$ & 1.33 & 5 & 0.08 \\
128 & $3.56-4$ & 1.19 & $1.50-3$ & 1.04 & 5 & 0.15 \\
256 & $1.60-4$ & 1.15 & $8.49-4$ & 0.82 & 5 & 0.45 \\
512 & $7.23-5$ & 1.15 & $5.18-4$ & 0.71 & 6 & 1.84 \\
1024 & $3.24-5$ & 1.16 & $3.24-4$ & 0.68 & 6 & 7.62 \\
\hline
\end{tabular}

For computation, we chose a family of trial spaces $W_{h}$ spanned by piecewise linear continuous trial functions $(\mu=1)$ with respect to uniform triangulations of $\Gamma$ with $N$ boundary elements independent of the Newton iteration. As stopping criteria of the Newton scheme, we used a relative residual reduction of $\varepsilon_{N}=10^{-8}$. We used the previous Newton iterate approximate solution $\hat{u}_{h}^{k}$ as an initial guess for the iterative solution of the linear system in the $(k+1)$-th step.

We considered the Laplace equation in the $L$ shaped domain described by the nodes $(0,0),(0.25,0),(0.25,0.25),(-0.25,0.25),(-0.25,-0.25)$ and $(0,-0.25)$ with the nonlinear boundary condition

$$
\frac{\partial}{\partial n} u(x)+u^{4}(x)=f(x)
$$

and $f$ was chosen in such a way that the exact solution is

$$
u(x)=-\log \left|x-x^{*}\right|, \quad x^{*}=(0.3,-0.3) .
$$

We chose the initial guess of the Newton iteration to be $u^{0}(x)=1$.

The Steklov-Poincaré operator formulation. The trial space $Z_{h}$ to approximate the Steklov-Poincaré operator $\tilde{S}$ as described in (2.8) was chosen to be spanned by piecewise constant trial functions, i.e., $\nu=0$ (see (2.6)). For inverting the discrete single layer potential matrix $V_{h}$ we used an inner conjugate gradient scheme with a preconditioner proposed in 21] where the stopping criteria was a relative error reduction of $\varepsilon_{C G}=10^{-8}$. Since the solution is regular, we expected the convergence rate 2 when measuring the error in $L^{2}(\Gamma)$ due to (2.26) and 1.5 in $H^{1 / 2}(\Gamma)$ due to (2.24), and they are substantiated in Table 1.

The indirect hypersingular integral formulation. Since $\Omega$ is a polygonal bounded domain, we get $v \in H^{1 / 2+s}(\Gamma), s<\sigma_{0}$ (see Remark 2.2 in [12]) with $\sigma_{0}=2 / 3$. Therefore the rate of convergence of the indirect hypersingular integral formulation is about $2 / 3$ in $H^{1 / 2}(\Gamma)$ and about $7 / 6$ in $L^{2}(\Gamma)$. This is reflected in 
TABLE 3. Hybrid solution strategy

\begin{tabular}{|c|c|c|c|c|c|c|}
\hline $\mathrm{N}$ & $\left\|u-u_{h}\right\|_{L^{2}(\Gamma)}$ & order & $\left\|u-u_{h}\right\|_{H^{1 / 2}(\Gamma)}$ & order & Iter & sec \\
\hline 32 & $1.35-3$ & & $7.42-3$ & & 6 & 0.11 \\
64 & $2.87-4$ & 2.23 & $2.40-3$ & 1.63 & 6 & 0.31 \\
128 & $6.43-5$ & 2.16 & $8.08-4$ & 1.57 & 6 & 0.85 \\
256 & $1.50-5$ & 2.10 & $2.78-4$ & 1.54 & 6 & 2.98 \\
512 & $3.61-6$ & 2.05 & $9.67-5$ & 1.52 & 7 & 12.44 \\
1024 & $8.86-7$ & 2.03 & $3.39-5$ & 1.51 & 7 & 49.34 \\
\hline
\end{tabular}

Table 2. Compared with the Steklov-Poincaré operator formulation, the computing times in Table 2 are significantly less, but we get less order of convergence.

The hybrid solution strategy. After computing the solution by the indirect hypersingular integral formulation, we used the resulting solution as an initial guess for the Steklov-Poincaré operator formulation. Just one such post-processing step yields a much faster solution with the order of convergence of the Steklov-Poincaré operator formulation, as seen in Table 3 .

\section{ACKNowledgments}

We would like to thank Dr. W. McLean and Dr. T. Tran for useful discussions and the referees for valuable comments and suggestions leading to many improvements.

\section{REFERENCES}

[1] K. E. Atkinson, G. A. Chandler, BIE methods for solving Laplace's equation with nonlinear boundary conditions. Math. Comp. 55 (1990) 451-457. MR 91d:65181

[2] O. Axelsson, Iterative Solution Methods. Cambridge University Press, 1994. MR 95f:65005

[3] R. Bialecki, A. J. Nowak, Boundary value problems in heat conduction with nonlinear material and nonlinear boundary conditions. Appl. Math. Model. 5 (1981) 417-421.

[4] C. Carstensen, M. Kuhn, U. Langer, Fast parallel solvers for symmetric boundary element domain decomposition equations. Numer. Math. 79 (1998) 321-347. MR 99d:65336

[5] P. G. Ciarlet, The Finite Element Method for Elliptic Problems. North-Holland, 1978. MR 58:25001

[6] M. Costabel, Boundary integral operators on Lipschitz domains: Elementary results. SIAM J. Math. Anal. 19 (1988) 613-626. MR 89h:35090

[7] R. L. Doucette, A collocation method for the numerical solution of Laplace's equation with nonlinear boundary conditions on a polygon. SIAM J. Numer. Anal. 30 (1993) 717-732. MR 94f:65113

[8] C. Eck, O. Steinbach, W. L. Wendland, A symmetric boundary element method for contact problems with friction. Math. Comput. Simulation 50 (1999) 41-59. CMP 2000:03

[9] P. P. B. Eggermont, J. Saranen, $L^{p}$ estimates of boundary integral equations for some nonlinear boundary value problems. Numer. Math. 58 (1990) 465-478. MR 91m:65325

[10] M. Ganesh, A BIE method for a nonlinear BVP. J. Comput. Appl. Math. 45 (1993) 299-308. MR 94f:65116]

[11] M. Ganesh, I. G. Graham and J. Sivaloganathan, A pseudospectral three-dimensional boundary integral method applied to a nonlinear model problems from finite elasticity. SIAM J. Numer. Anal. 31 (1994) 1378-1414. MR 95g:45011

[12] M. Ganesh, O. Steinbach, Nonlinear boundary integral equations for harmonic problems. J. Int. Equations. Appl. 11(4) (1999).

[13] G. C. Hsiao, W. L. Wendland, The Aubin-Nitsche Lemma for integral equations. J. Int. Equations. Appl. 3 (1981) 299-315.

[14] F. Incropera, D. DeWitt, Fundamentals of Heat and Mass Transfer, John Wiley \& Sons, New York, 1990. 
[15] D. B. Ingham, P. J. Heggs, M. Manzoor, Boundary integral equation solution of nonlinear plane potential problem. IMA J. Numer. Anal. 1 (1981) 415-426. MR 83a:65122

[16] M. A. Kelmanson, Solution of nonlinear elliptic equations with boundary singularities by an integral equation method. J. Comp. Phys. 56 (1984) 244-258.

[17] K. Ruotsalainen, J. Saranen, On the collocation method for a nonlinear boundary integral equation. J. Comput. Appl. Math. 28 (1989) 339-348. MR 91d:65180

[18] K. Ruotsalainen, W. L. Wendland, On the boundary element method for some nonlinear boundary value problems. Numer. Math. 53 (1988) 299-314. MR 89h:65189

[19] Y. Saad, Iterative Methods for Sparse Linear Systems. PWS, Boston, 1996.

[20] A. H. Schatz, V. Thomée, W. L. Wendland, Mathematical Theory of Finite and Boundary Element Methods. Birkhäuser, Basel, 1990. MR 92f:65004

[21] O. Steinbach, W. L. Wendland, The construction of some efficient preconditioners in the boundary element method. Adv. Comput. Math. 9 (1998) 191-216. MR 99j:65219

School of Mathematics, University of New South Wales, Sydney 2052, Australia

E-mail address: ganesh@maths.unsw.edu.au

Mathematisches Institut A, Universität Stuttgart, Pfaffenwaldring 57, 70569 StuttGART, Germany

E-mail address: steinbach@mathematik.uni-stuttgart.de 